\title{
ON ENTIRE FUNCTIONS OF AFFINE LINEAGE
}

\author{
WOLFGANG DAHMEN AND CHARLES A. MICCHELLI
}

\begin{abstract}
In this paper we provide a characterization of entire functions whose zero set is a union of real hyperplanes.
\end{abstract}

The purpose of this note is to establish a new property of entire functions of several complex variables whose zero sets are a union of hyperplanes. Our interest in this subject arose from questions concerning limits of multivariate $B$-splines and it is our intention to present this relationship elsewhere [2].

We will say that an entire function $f(z), z \in \mathbf{C}^{s}(f \neq 0)$ has affine lineage if its zero set $\left\{z \mid f(z)=0, z \in \mathbf{C}^{\mho}\right\}$ consists of a union of hyperplanes $H=\{z \mid z \cdot \zeta=t$, $\left.z \in \mathbf{C}^{s}\right\}$ where $\zeta \in \mathbf{C}^{s}-\{0\}, t \in \mathbf{R}$, and $z \cdot \zeta=z_{1} \bar{\zeta}_{1}+\cdots+z_{s} \bar{\zeta}_{s}, \quad z=$ $\left(z_{1}, \ldots, z_{s}\right), \zeta=\left(\zeta_{1}, \ldots, \zeta_{s}\right)$. When all the hyperplanes in the zero set of $f$ are determined by real vectors, $\zeta \in \mathbf{R}^{s}$, we say that $f$ has real affine lineage. The reason for this terminology comes from a theorem of Motzkin and Schoenberg [1], which characterizes functions of affine lineage as limits of products of affine functions, $a(z)=z \cdot \zeta-t$.

Our main theorem below gives a completely different criterion for a function to have real affine lineage. In the sense described below the requirement of having real affine lineage is shown to be intrinsically univariate in nature.

THEOREM. Let $f$ be an entire function on $\mathbf{C}^{s}$. Then $f$ has real affine lineage if and only if, for every $x, y \in \mathbf{R}^{s}, f(x+z y), z \in \mathbf{C}$, has only real zeros or is identically zero.

Thus, in particular, if $P(x)$ is a polynomial on $\mathbf{C}^{s}$ such that for all $x, y \in \mathbf{R}^{s}$ the univariate polynomial $P(x+z y)$ has only real zeros then $P$ has real affine lineage. Hence according to the Weierstrass factorization theorem from [1], $P$ admits a global factorization as a product of affine functions,

$$
P(x)=\tau\left(y_{1} \cdot x-t_{1}\right) \cdots\left(y_{n} \cdot x-t_{n}\right), \quad y_{j} \in \mathbf{R}^{s}, t_{j} \in \mathbf{R}, \tau \in \mathbf{C} .
$$

Proof OF THEOREM. Suppose $f$ has real affine lineage and $f(x+z y)$ has a complex zero, say $z_{0} \in \mathbf{C}$, for $x, y \in \mathbf{R}^{s}$. Then there is a real hyperplane $H=\{z \mid \zeta \cdot$ $z-t=0\}, \zeta \in \mathbf{R}^{s}, t \in \mathbf{R}$, passing through $z_{0}=x+z_{0} y$ and lying entirely in the zero set of $f$. Since $\operatorname{Im} z_{0} \neq 0$ it immediately follows that $x+t y$ is in $H$ for all

Received by the editors March 19, 1981 and, in revised form, June 6, 1981.

1980 Mathematics Subject Classification. Primary 32A15. 
$t \in \mathbf{R}$. Hence $f(x+z y) \equiv 0$ and so the necessity of our condition is verified. The sufficiency of our characterization will be proved next.

First, let us observe that it suffices to assume $f$ is real on the real axis, since otherwise we can apply our argument below to $\overline{f(\bar{z})} f(z)$. Now, suppose $f\left(z_{0}\right)=0$ and $z_{0}=x_{0}+i y_{0}, x_{0}, y_{0} \in \mathbf{R}^{s}$. We will find a real hyperplane in the zero set of $f$ passing through $z_{0}$. To this end, observe that $f\left(x_{0}+z y_{0}\right) \equiv 0$ for $z \in \mathbf{C}$ due to the hypothesis on $f$. Thus $f\left(x_{0}\right)=0$ and so $f$ admits a factorization $f=p_{1} \cdots p_{m} \Omega$ (in the ring of functions which are analytic in a neighborhood of $x_{0}$ ) where each $p_{j}$ is a (real) irreducible distinguished polynomial and $\Omega$ is a unit. Let us assume for definiteness that $p=p_{1}$ vanishes on $x_{0}+t y_{0}$, for $t$ in some real neighborhood of the origin. Thus $\nabla p\left(x_{0}+t y_{0}\right) \cdot y_{0}=0$ for all $t$ in this neighborhood. Moreover, since $p$ is irreducible it is easy to see that for some $t_{0}$ near zero $\nabla p\left(x_{0}+t_{0} y_{0}\right) \neq 0$. Let us introduce $h(z)=p\left(z+w_{0}\right)$ where $w_{0}=x_{0}+t_{0} y_{0}$. Thus $h(0)=0$ and $\nabla h(0) \neq 0$. We will now prove the following property of $h$. All real vectors $y \in \mathbf{R}^{s}$ satisfying $\nabla h(0) \cdot y=0$ are zeros of $h$, i.e., $h(y)=0$. Assuming this fact for the moment, it quickly follows that $H=\left\{z \mid \nabla p\left(w_{0}\right) \cdot\left(z-w_{0}\right)=0\right\}$ is the real hyperplane which we seek. To see this, observe that if $\nabla p\left(w_{0}\right) \cdot\left(z-w_{0}\right)=0$ we have $\nabla h(0) \cdot\left(\operatorname{Re} z+t \operatorname{Im} z-w_{0}\right)=0$, all $t \in \mathbf{R}$. Hence $h\left(\operatorname{Re} z+t \operatorname{Im} z-w_{0}\right)=$ $p(\operatorname{Re} z+t \operatorname{Im} z)=0$ and consequently $f(z)=0$. In the same manner, we observe that $z_{0} \in H$. Thus it remains to prove our assertion concerning $h$, that is, every real vector $y$ in the domain of $h$ satisfying $\nabla h(0) \cdot y=0$ is a zero of $h$.

For ease of notation, we set $\zeta=\nabla h(0)$ and suppose to the contrary that there is a $y \in \mathbf{R}^{s}$ with $\zeta \cdot y=0$ while $h(y) \neq 0$. We define $F(t, z)=h(t \zeta+z y)$ and observe that $F(0,0)=0$ and $F(0, z)=h(z y) \neq 0$. Therefore the Weierstrass preparation theorem implies that there is an integer $m \geqslant 1$ and a neighborhood $U_{2}$ of the origin in $\mathbf{C}^{2}$ such that on $U_{2}$

$$
F(t, z)=\left(z^{m}+A_{1}(t) z^{m-1}+\cdots+A_{m-1}(t) z+A_{m}(t)\right) \cdot \Omega(t, z)
$$

where each $A_{j}$ is analytic in a neighborhood $U_{1}$ of the origin in $\mathbf{C}, A_{j}(0)=0$ and $\Omega(0,0) \neq 0$. Since

$$
0<\|\zeta\|^{2}=F_{1}(0,0)=A_{m}^{\prime}(0) \Omega(0,0)
$$

we have $A_{m}(t)=d t+\cdots$ where $d \neq 0$. We choose a sign $\delta= \pm 1$ so that $\operatorname{Re} \delta d>0$. Let $d^{\prime}=\delta d$ and observe that $F\left(\delta t^{m}, t z\right)=t^{m} Q(t, z) P(t, z)$ where $Q(t, z)=z^{m}+B_{1}(t) z^{m-1}+\cdots+B_{m-1}(t) z+B_{m}(t)$, with each $B_{j}$ analytic in $U_{1}, B_{m}(0)=d^{\prime}, B_{j}(0)=0, j \leqslant m-1$, and $P(0,0) \neq 0$. Notice that $m>2$ because $F_{2}(0,0)=\zeta \cdot y=0$. Consequently, since $Q(0, z)=z^{m}+d^{\prime}$ we conclude there exist a $\tau \in \mathbf{R}, z_{0} \in \mathbf{C}-\mathbf{R}$ with $F\left(\delta \tau^{m}, \tau z_{0}\right)=0$. Thus along the line $\delta \tau^{m} \zeta+\tau z y, h$ has a complex zero at $z=z_{0}$. This contradiction completes the proof of our assertion concerning $h$ and also the theorem.

Further discussion of entire functions having affine lineage can be found in [3]. 


\section{REFERENCES}

1. T. S. Motzkin and I. J. Schoenberg, On lineal entire functions of $n$ complex variables, Proc. Amer. Math. Soc. 3 (1952), 517-526.

2. W. Dahmen and C. A. Micchelli, On limits of multivariate B-splines, J. Analyse Math. (to appear).

3. $\ldots$, On entire functions of affine lineage, Report No. 8324, IBM Research Center, Yorktown Heights, N. Y., 1980.

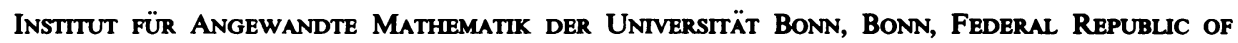
Germany

IBM Research Center, P. O. BoX 218, Yorktown Heights, New York 10598 